\title{
Effect of Magnetic Field on Flow in a Porous Medium over a Permeable Stretching Wall in the Presence of Thermal Radiation and Suction/Injection
}

\author{
Sahar M. Abdel-Gaied \\ Department of Science and Mathematics, Faculty of Education, Assiut University, the New Valley 72111, Egypt.
}

\begin{abstract}
A study has been carried out to analyze the effect of Magnetic field flow in a porous medium with consider thermal radiation and suction/injection over a permeable stretching wall. We consider wall boundary conditions are distribution of wall temperature, in addition to momentum, both first and second laws of the thermodynamics analyses of the problem are investigated. A set of solution to the non-linear equations is presented using the shooting method. A parametric study showing the effects of magnetic parameter M; thermal radiation parameter $R$ and the injection parameter $f_{w}$. The effect of various parameter on the fluid flow and heat transfer profile on the velocity and temperature profile as well as the wall heat transfer are presented graphically and in tabulated.
\end{abstract}

Keyword: Magnetic Field, Thermal Radiation, Stretching wall, Porous Medium.

\section{Introduction}

The study of magnet hydrodynamic (MHD) over a porous stretching sheet has gained considerable attention due to its applications in the industry, which the use of magnetic field that influences heat generation/absorption process in electrically conducting fluid flows have important engineering applications, for example in many metallurgical processes such as drawing of continuous filaments through quiescent fluids, and annealing and tinning of copper wires. The study of free convective mass transfer effects are dominate feature in many engineering applications such as rocket nozzles, cooling of nuclear reactors, high rinks in turbine blades, high speed aircrafts and their atmospheric reentry. So, in this years, progress has been considerably made in the study of heat and mass transfer in magneto hydrodynamic flows due to its application in many device, like the MHD power generator and hall accelerator. The influence of magnetic field on the flow of an electrically can ducting viscous fluid with mass transfer and radiation absorption is also useful in planetary atmosphere research. Sparrow and Cess [1] studied the effect of magnetic fields on natural convection heat transfer. The Ram [2] considered the effect of magnetic fields on both natural and forced convection heat transfer problems .

Kinyanjui et. Al [3] analyzed simultaneous heat and mass transfer in unsteady free convection flows with radiation absorption past an impulsively started infinite vertical porous Plate Subjected to a strong magnetic field Afifi [4] had studied effects of temperature - dependent viscosity with sort and Dufour effects on non - Darcy MHD free convective heat and mass transfer of a viscous, incompressible and electrically conducting fluid Past a vertical isothermal surface embedded in a saturated porous medium Hay at et al. [5] considered the MHD flow over a nonlinearly stretching sheet by employing the modified Adomain decomposition method. On the other side, radiation feat transfer effects from a porous wall on forced convection flow are very important in technology and high temperature processes, and very little is know $\mathrm{n}$ about the effects of recitation on the boundary layer flow of radiating fluid past body. The problem of radiation and mass transfer effects on MHD free convection flow past an impulsively started isothermal vertical plate with dissipation was examined by Suneetha[6]. Abd El - Aziz [7] has investigated the problem of thermal radiation effects on magneto hydrodynamic mixed convection flow of micro polar fluid Past a continually moving semi - infinite Plate for high temperature difference. Mohamed and Abo - Dahab [8] present the effects of chemical resection boundary by a semi - infinite vertical porous plate in the presence of heat generation.

Heat and mass transfer problems with boundary layer flow cased by a continuously stretching sheet is often encountered in many engineering and industrial processes because a numerous applications in geophysics and energy related engineering problems that include both metal and Polymer sheets. The problem of heat and mass transfer on a stretching sheet with Suction or blowing was considered by Gupta and Gwpta [9].

Many works have been reported on the flow with heat and mass transfer over a stretched Surface in the presence of a magnetic field with various aspects [10 - 15]. In this present work deals with magnetic field flow over of permeable stretching wall in presence of thermal radiation and suction and injection that flow in a porous medium. These equations are solved numerically using shooting and Rung-kutta method. The effects of temperature and viscosity parameter, suction / injection, Prandtl number, radiation and magnetic field are investigated and analyzed with the help of their graphical representations. 


\section{Analysis}

Consider the steady two-dimensional flow through a homogenous porous medium of magnetic field with consider thermal radiation and suction/injection, over a stretching wall. The transport properties of medium can be considered. The origin is kept fixed while the wall is stretching and the y-axis is perpendicular to the surface. Using the above-mentioned assumptions, the continuity equation is,

$$
\begin{aligned}
& \frac{\partial u}{\partial x}+\frac{\partial v}{\partial y}=\mathbf{O}, \\
& \rho\left(u \frac{\partial u}{\partial x}+v \frac{\partial u}{\partial y}\right)=\mu_{e f f} \frac{\partial^{2} u}{\partial y^{2}}-\left(\frac{\mu}{K}+\sigma B_{0}{ }^{2}\right) u \\
& u \frac{\partial T}{\partial x}+v \frac{\partial T}{\partial y}=\alpha \frac{\partial^{2} T}{\partial x^{2}}-\frac{\partial q_{r}}{\partial y} .
\end{aligned}
$$

Where $u$ and $v$ are the velocity components along $x$ and $y$ coordinates, respectively, $T$ is temperature, $K$ is the permeability, $\rho$ is the density, $\alpha$ is the effective thermal diffusivity, $T_{w}$ is the surface temperature, $T_{\infty}$ is the free stream temperature and $q_{r}$ is the radiative heat flux.

The appropriate boundary conditions are given by:

$$
\begin{aligned}
& u(x, 0)=c x, v(x, 0)=v_{w}, u(x, \infty)=0, \\
& T(x, 0)=T_{\infty}+T_{0}(x)^{n}, T(x, \infty)=T_{\infty} ; \text { or } \\
& -k \frac{\partial T}{\partial y}=q_{0}(x)^{n}, T(x, \infty)=T_{\infty} .
\end{aligned}
$$

where $v_{w}$ is the injection velocity, and $q_{0}$ wall heat flux coefficient.

The continuity equation is automatically satisfied by defining a stream function $\psi(x, y)$ such that:

$$
u=\frac{\partial \psi}{\partial y} \text { and } v=-\frac{\partial \psi}{\partial x} \text {. }
$$

Equations. (1-4) are now non-dimensionalized using the following similarity transformation:

$$
\eta=\frac{y}{\sqrt{K}}, \quad \psi=c x \sqrt{K} f(\eta) \text {. }
$$

Where $f$ is the dimensionless stream function, under the transformations in Eq. (6), the system of non-linear differential equations (1-4) becomes:

$$
\begin{aligned}
& f^{\prime \prime \prime}+\operatorname{Re}\left(f f^{\prime \prime}-f^{\prime 2}\right)-(1+\operatorname{Re} M) f^{\prime}=0 \\
& \theta^{\prime \prime}+\frac{\operatorname{Re} \cdot \operatorname{Pr}}{\left(1+N_{r}\right)} f \theta^{\prime}-n \frac{\operatorname{Re} \cdot \operatorname{Pr}}{\left(1+N_{r}\right)} f^{\prime} \theta=0
\end{aligned}
$$

With the boundary conditions

$$
f^{\prime}(0)=1, f(0)=-\frac{v_{w}}{c \sqrt{K}}=f_{w}, f^{\prime}(\infty)=0
$$

Where Re, $M$ are the Reynolds number and the Magnetic field parameter, respectively

$$
\mathrm{Re}=\frac{\rho c K}{\mu}, M=\frac{\sigma B_{0}{ }^{2}}{\rho c}
$$

$f_{w}$ is the injection parameter, positive/negative values of $f_{w}$ show suction/injection into/from the porous surface.

- Thermal radiation analysis:

Introducing a similarity function $\theta$ as:

$$
T-T_{\infty}=T_{r e f}(x)^{n} \theta(\eta)
$$

Where $T_{r e f}$ is $T_{0}$ and $q_{0} \sqrt{K} / k$ for the power-law temperature and heat flux boundary condition, respectively:

$$
T=T_{r e f}(x)^{n} \theta(\eta)+T_{\infty}
$$

Where the radiative heat flux is 


$$
q_{r}=-\frac{4 \sigma}{3 \delta} \frac{\partial T^{4}}{\partial y}
$$

where $\sigma$ is the Stefan-Boltzman constant and $\delta$ is the Rosseland mean absorption coefficient. Assuming that the temperature differences within the flow are sufficiently small such that $T^{4}$ may expressed as a linear function of temperature:

$T^{4} \cong 4 T_{\infty}^{3} T-3 T_{\infty}^{4}$

where the higher-order terms of the expansion are neglected.

Where $\operatorname{Pr}=\frac{\mu}{\rho \alpha}$; the Prandtl number and $N_{r}=\frac{16 \sigma T_{\infty}^{3}}{3 \delta \alpha}$ is radiation parameter.

$$
\begin{aligned}
& \theta(0)=1, \theta(\infty)=0 \text {; for power- law temperature } \\
& \theta^{\prime}(0)=-1, \theta(\infty)=0 \text {; for power - law heat flux. }
\end{aligned}
$$

The local Nusselt number is given by:

$$
N u_{x}=\frac{h x}{k}=\left\{\begin{array}{lr}
-\frac{\theta^{\prime}(0) x}{\sqrt{K}} ; & \text { Power-lawtemperature } \\
\frac{q_{w} x}{k\left(T_{w}-T_{\infty}\right)}=\frac{x}{\theta(0) \sqrt{K}} & \text { Power -lawheat flux }
\end{array}\right.
$$

\section{Result and discussion}

Some numerical calculation have been carried out for the non-dimensional velocity $u$, temperature $t$, magnetic field $M$ and radiation, Fig. (1) appears the effect of magnetic field parameter on velocity which the velocity decrease with magnetic field increasing. The effects of magnetic field parameter on the temperature distribution while the temperature increase with the magnetic field parameter increment; it was studies in Fig. (2). The effect of Reynold number and magnetic field on skin fraction coefficient are reported in Fig. (3) which the skin fraction coefficient increases with increase the Reynold number and increase the magnetic field parameter. Fig(4) present the effect of Reynold number and magnetic field parameter on heat transfer rate which the heat transfer rate increase as we expect with increase of Reynold number and with decrease of magnetic field parameter. Suction/injection parameter on a heat transfer rate and effect of Magnetic field parameter show In Fig. (5) which the heat transfer rate increase with decrease of the magnetic field and suction/injection parameter increment. Fig. (6) present the effect of magnetic field parameter and power-law temperature o a heat transfer rate, which the heat transfer rate increase with power -law temperature increase and decrease of magnetic field parameter. The effect of Radiation parameter with Reynold number rate and with Prandtl number on heat transfer rate are show in Figs. (7-8) which the heat transfer rate increase with increasing of Reynold number and decrease of radiation parameter also it increase with the increment of parameter of Prandtl number and reduction of the Radiation parameter.

In the Fig. (9) we can show the Magnetic field parameter and suction/injection effect on the skin fraction coefficient where the skin fraction coefficient increase with increase of the magnetic field parameter and increase of suction/injection parameter. The temperature increases with the radiation parameter increases that appear in Fig. (10) in the other side the heat transfer rate increase with the power-law temperature increase ad with decrease of radiation parameter and the heat transfer rate increase with suction/injection parameter increase that are shown in Figs. (11).

\section{Reference}

[1] E.M. Sparrow and R.D. Cess, The effect of a magnetic field on free convection heat transfer, International Journal of Heat and Mass Transfer, 3, (1961) 267-274.

[2] H.S. Takhar and P.C. Ram, Magnetohydrodynamic free convection flow of water at $4{ }^{\circ} \mathrm{C}$ through a porous medium, International Communications in Heat and Mass Transfer, 21, (1994) 371-376.

[3] M. Kinyanjui, J.K. Kwanza, and S.M. Uppal, Magneto-hydrodynamic free convection heat and mass transfer of a heat generating fluid past an impulsively started infinite vertical porous plate with hall current and radiation absorption. Energy Conservation and Management 42, (2001) 917-931

[4] A.A. Afify, Effects of Temperature-Dependent Viscosity with Soret and Dufour Numbers on Non-Darcy MHD Free Convective Heat and Mass Transfer Past a Vertical Surface Embedded in a Porous Medium, Transport in Porous Media, 66, (2007) 391-401.

[5] T. Hayat, Q. Hussain and T. Javed, The modified decomposition method and Pad'e approximants for the MHD flow over a nonlinear stretching sheet, Nonlinear Analysis: Real World Applications, 10 (2009) 966-973 
[6] S. Suneetha , N Bhasker Reddy and V Ram Chandra Prasad Radiation and mass transfer effects on MHD free convection flow past an impulsively started isothermal vertical plate with dissipation. Thermal Science, Vol. 13, No.2, (2009). pp. 171-181

[7] M. Abd-El Aziz, Thermal radiation effects on magnetohydrodynamic mixed convection flow of a micropolar fluid past a continuously moving semi-infinite plate for high temperature differences, Acta Mechanica, 187, (2006) 113-127.

[8] R.A. Mohamed and S.M. Abo-Dahab, Influence of chemical reaction and thermal radiation on the heat and mass transfer in MHD micropolar flow over a vertical moving porous plate in a porous medium with heat generation, 48, (2009) 1800-1813.

[9] P.S. Gupta and A.S. Gupta, Heat and mass transfer on a stretching sheet with suction or blowing, Can. J. Chem. Eng., 55, (1977) $744-746$.

[10] R. Kandasamy, K. Periasamy and K.K. Sivagnana Prabhu, Chemical reaction, heat and mass transfer on MHD flow over a vertical stretching surface with heat source and thermal stratification effects, International Journal of Heat and Mass Transfer 48 (2005) 4557-4561.

[11] I-Chung Liu, Flow and heat transfer of an electrically conducting fluid of second grade in a porous medium over a stretching sheet subject to a transverse magnetic field Original Research Article, International Journal of Non-Linear Mechanics, 40, (2005) 465474.

[12] M. Abd El-Aziz and A.M. Salam, MHD-mixed convection and mass transfer from a vertical stretching sheet with diffusion of chemically reactive species and space or temperature dependent heat source. Can. J. Phys., 85, (2007) 359-373.

[13] A.M. Salem, M. Abd El-Aziz, Effect of Hall currents and chemical reaction on hydromagnetic flow of a stretching vertical surface with internal heat generation/absorption, Applied Mathematical Modelling, 32, (2008)1236-1254.

[14] M.F. El-Amin, Combined effect of internal heat generation and magnetic field on free convection and mass transfer flow in a micropolar fluid with constant suction, Journal of Magnetism and Magnetic Materials, 270, (2004) 130-135.

[15] C.H. Chen, Combined heat and mass transfer in MHD free convection from a vertical surface with Ohmic heating and viscous dissipation, International Journal of Engineering Science, 42, (2004)699-713.

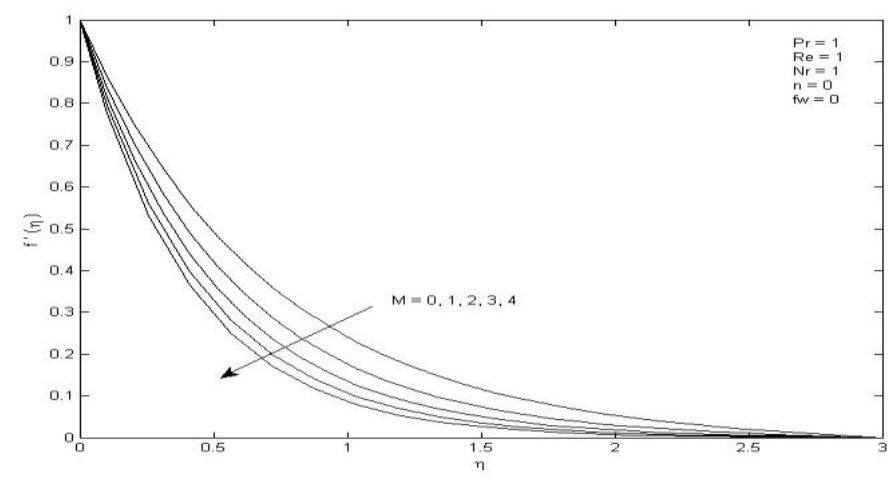

Fig(1) Velocity profiles for various values of magnetic parameter $\mathrm{M}=0,1,2,3,4 . \mathrm{M}=0,1,2,3,4$.

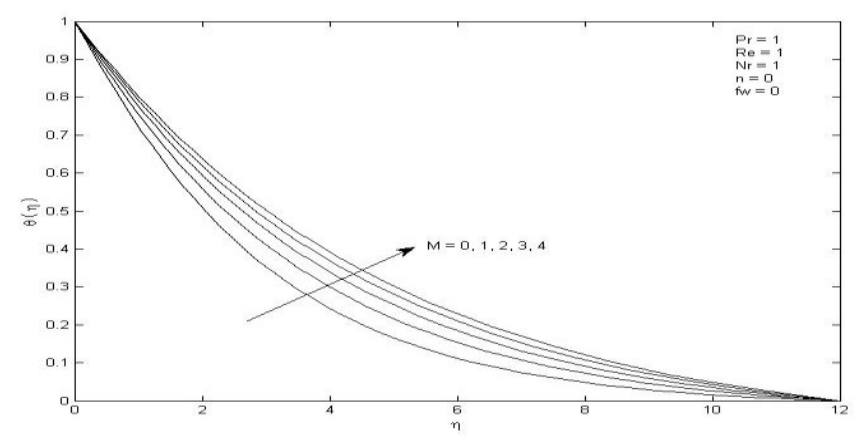

Fig(2) Temperature profiles for various values of magnetic parameter M. $=0,1,2,3,4$.

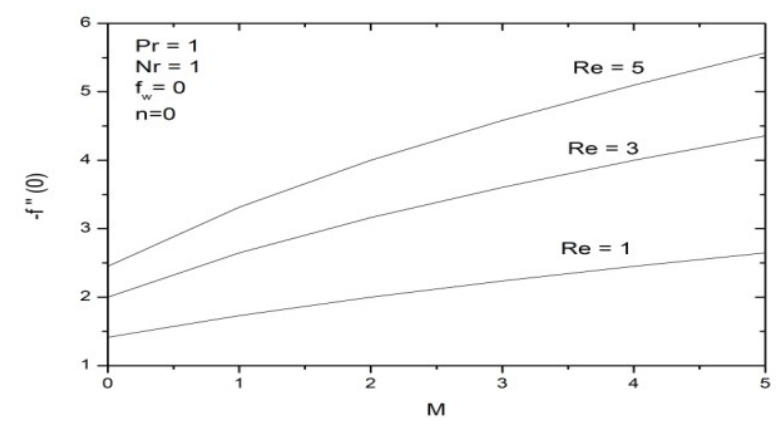


Fig(3 Dimensionless wall velocity gradient $\mathrm{f}^{\prime}(0)$ as a function of magnetic parameter $\mathrm{M}$ for various values of Reynolds number $\mathrm{Re}=1,3,5$

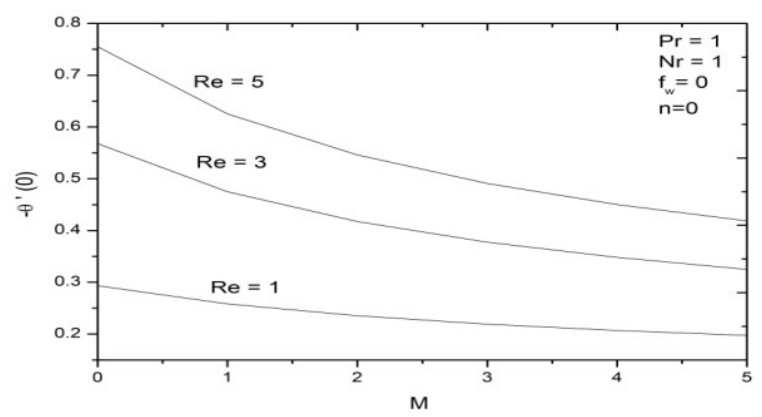

Fig(4) Dimensionless wall temperature gradient $-\theta \overline{(0)}$ as a function of magnetic parameter $\mathrm{M}$ for various values of Reynolds number $\mathrm{Re}=1,3,5$

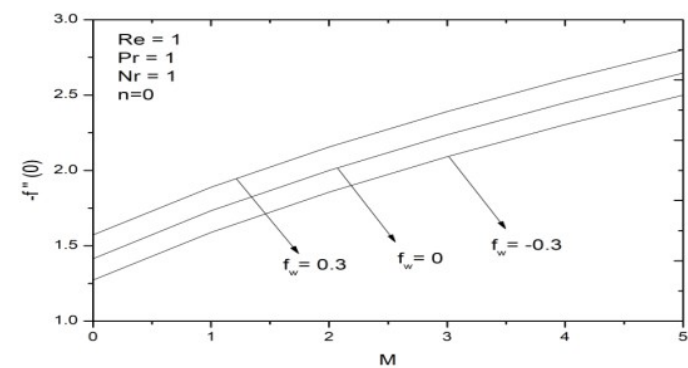

Fig(5) Theta Dimensionless wall velocity gradient $f^{\prime \prime}(0)$ as a function of magnetic parameter $M$ for various values of suction/injection parameter $\mathrm{f}_{\mathrm{w}}=-0.3,0,0.3$

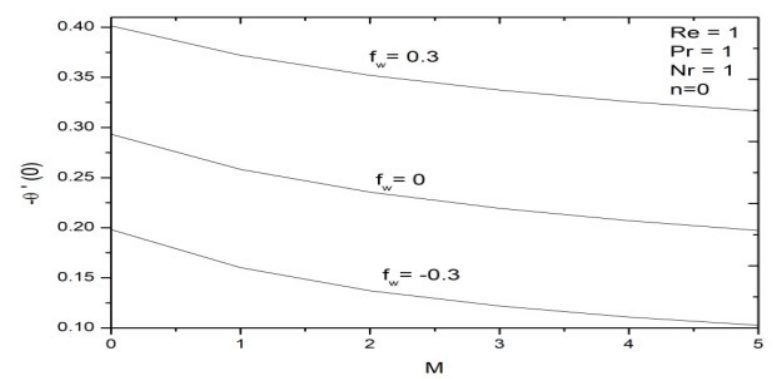

Fig(6) Dimensionless wall temperature gradient $-\theta \overline{(0)}$ as a function of magnetic parameter $M$ for various values of suction/injection parameter $\mathrm{f}_{\mathrm{w}}$

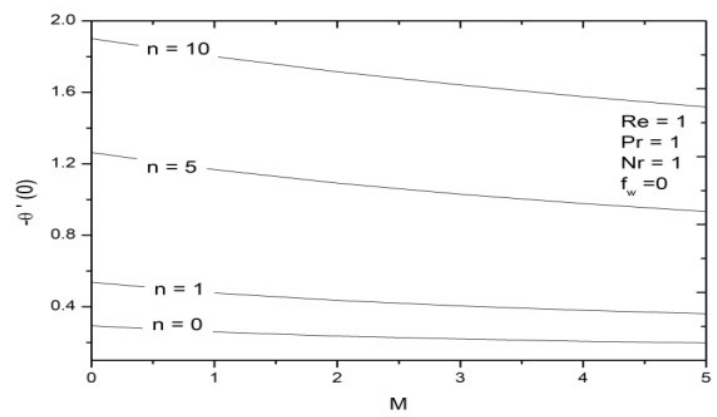

Fig(7) Dimensionless wall temperature gradient $-\theta-(0)$ as a function of magnetic parameter $M$ for various values of suction/injection parameter $f_{w}$ 


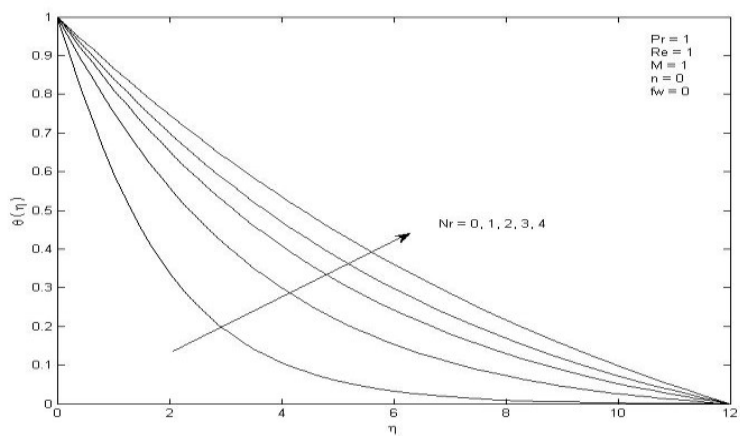

Fig(8) Temperature profiles $\theta(\eta)$ for various values of radiation parameter $\mathrm{Nr}$.

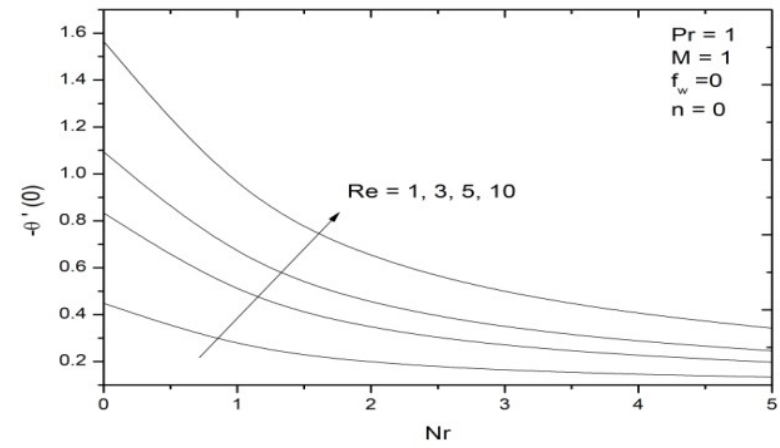

Fig(9) Dimensionless wall temperature gradient $-\theta \overline{(0)}$ as a function of radiation parameter Nr for various values of Reynolds number Re.

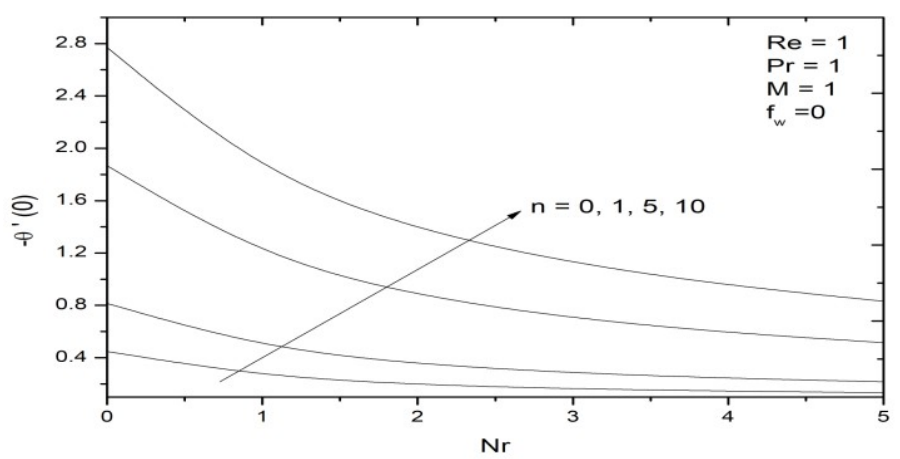

Fig(10) Dimensionless wall temperature gradient $-\theta \overline{(0)}$ as a function of radiation parameter $\mathrm{Nr}$ for various values of power law index parameter $n$.

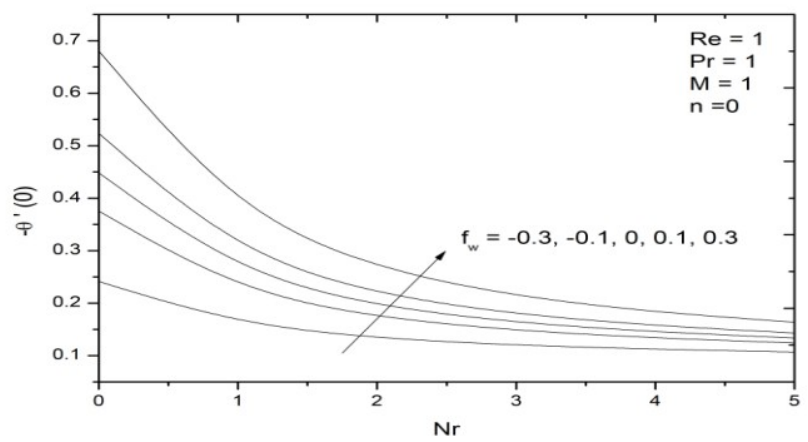

Fig(11) Dimensionless wall temperature gradient $-\theta \overline{(0)}$ as a function of radiation parameter Nr for various values of suction/injection parameter $f_{w}$. 